\title{
Isolation and characterization of biliary epithelial and stromal cells from resected human cholangiocarcinoma: A novel in vitro model to study tumor-stroma interactions
}

\author{
MARCO MASSANI $^{1}$, TOMMASO STECCA ${ }^{1}$, LUCA FABRIS $^{3}$, EZIO CARATOZZOLO ${ }^{1}$, CESARE RUFFOLO $^{1}$, \\ ALBERTO FURLANETTO $^{2}$, STUART MORTON ${ }^{3,4}$, MASSIMILIANO CADAMURO ${ }^{4}$, \\ MARIO STRAZZABOSCO ${ }^{5}$ and NICOLÒ BASSI ${ }^{1}$
}

\begin{abstract}
${ }^{1}$ IV Department of Surgery, Regional Center for HPB Surgery, and ${ }^{2}$ Department of Pathological Anatomy, Regional Hospital of Treviso, Treviso; ${ }^{3}$ Department of Surgical and Gastroenterological Sciences, University of Padova, Padova; ${ }^{4}$ Department of Surgical and Gastroenterological Sciences, University of Milan-Bicocca, Milan, Italy; ${ }^{5}$ Department of Medicine and Liver Center, Yale University, New Haven, CT, USA
\end{abstract}

Received March 6, 2013; Accepted April 28, 2013

DOI: $10.3892 / o r .2013 .2568$

\begin{abstract}
Cholangiocarcinoma (CCA) is a devastating malignancy arising from the bile ducts. Cancer-associated fibroblasts (CAFs) are key players in CCA invasiveness and in the generation of a desmoplastic reaction. The aim of the present study was to develop a novel model by which to study tumor-stroma interactions using primary cultures of human biliary epithelial cells (hBECs) and stromal cells (SCs) in CCA. hBECs and SCs, isolated from surgical resections $(n=10)$, were semi-purified by centrifugation on a Percoll gradient; hBECs were further immunopurified. hBECs and SCs were characterized using epithelial [cytokeratin 7 (CK7) and CK19] and mesenchymal [vimentin (VMN), $\alpha$-smooth muscle actin ( $\alpha$-SMA), CD68] cell markers. The purity of cultured cells was assessed by fluorescent immunocytochemistry. hBECs were HEA125/CK7/ CK19-positive and VMN/ $\alpha$-SMA-negative. SCs were VMN/ $\alpha$ SMA-positive and CK7/CK19-negative. CCA 2-D culture models have been described but they use long-standing CCA cell lines of various biliary tumor cell origins with stromal cells derived from non-cholangiocarcinoma tissues. Recently, a novel 3-D organotypic co-culture model of rat cholangiocarcinoma was described. In the present study, we obtained
\end{abstract}

Correspondence to: Dr Marco Massani, IV Department of Surgery, Regional Center for HPB Surgery, Regional Hospital of Treviso, Piazza Ospedale, I-31100 Treviso, Italy

E-mail:mmassani@ulss.tv.it

Abbreviations: $\alpha$-SMA, $\alpha$-smooth muscle actin; CAF, cancerassociated fibroblast; CCA, cholangiocarcinoma; CK7, cytokeratin-7; CK19, cytokeratin-19; ICC, intrahepatic cholangiocarcinoma; hBEC, human biliary epithelial cell; SC, stromal cell; VMN, vimentin

Key words: cell isolation, cholangiocarcinoma, tumor-stroma interactions, stromal cells pure and stable primary cultures of hBECs and SCs from CCA surgical specimens. These cell cultures may provide a useful tool by which to study CCA tumor-stroma interactions.

\section{Introduction}

Cholangiocarcinoma (CCA) is a rare and devastating malignancy arising from the epithelium of the bile ducts (1). Patient overall survival at 5 years from diagnosis is $<5 \%$ (2). CCA epidemiology is shifting towards a younger population, i.e. $<65$ years of age, with an increasing incidence of intrahepatic CCA and an evidently decreasing rate of extrahepatic CCA (3). Currently, surgery is the only curative therapy available for CCA patients, and palliation is a valuable aid in relieving symptoms of obstructive jaundice and recurrent cholangitis $(4,5)$.

Tumor progression has been recognized as the consequence of a dynamic 'crosstalk' between different cell types of the tumor parenchyma and the surrounding tissue, the tumor stroma $(6,7)$. Dissimilarities between a 'normal' and a 'reactive' stroma are currently the subject of detailed studies (5,8-10). The normal stroma in many organs contains a minimal amount of fibroblasts relative to the extracellular matrix. The reactive stroma, however, is associated with an increase in fibroblasts, capillary density and deposition of fibrin and collagen type-1 (7).

Cancer cells may alter the adjacent stroma to create a microenvironment that permits and supports tumor growth. Morphological evidence describes it as a desmoplastic reaction involving many cell types, including endothelial cells and their precursors, pericytes, smooth muscle cells, cancerassociated fibroblasts (CAFs), myofibroblasts, neutrophils, eosinophils, basophils, mast cells, T and B lymphocytes, macrophages and dendritic cells (11). Endothelial cells, macrophages and CAFs play a key role in promoting growth and tumor progression (12). 


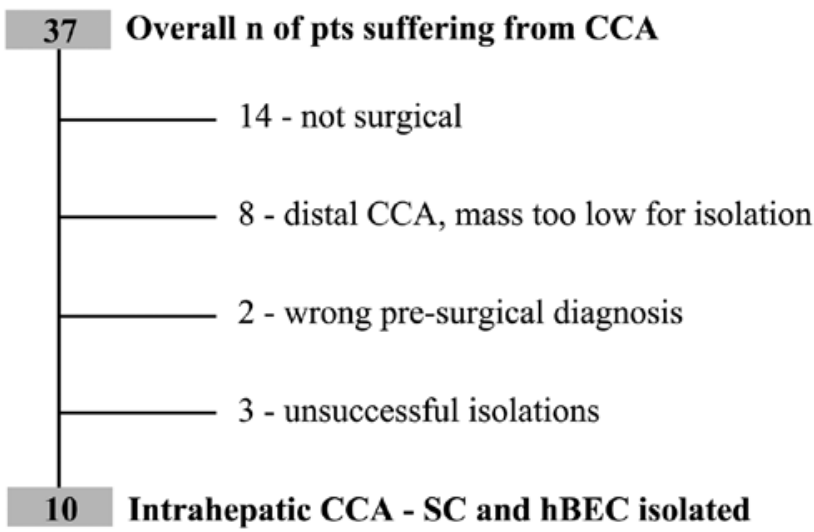

Figure 1. Flow chart of cholangiocarcinoma patient selection. Between 2010 and 2012, 37 patients were diagnosed with cholangiocarcinoma (CCA) at Treviso Hospital; 10 patients were included in the study. SC, stromal cell; hBEC, human biliary epithelial cell.

Compared with fibroblasts present in a quiescent state in connective tissues or activated in response to tissue damage or organ fibrosis, CAFs are not susceptible to apoptosis and as such, are perpetually activated and do not return to a normal phenotype. Morphologically, CAFs are elongated mesenchymal cells positive for $\alpha$-smooth muscle actin ( $\alpha$-SMA), fibroblast activation protein (FAP), Thy-1, desmin and the S100A4 protein (13). Results of several in vivo and in vitro studies indicate that CAFs promote tumor progression both in a proliferative and an invasive manner through the secretion of various growth factors and the activation of numerous intracellular signaling pathways (14).

The desmoplastic stroma of intrahepatic cholangiocarcinoma (ICC) is rich in $\alpha$-SMA-positive fibroblasts that surround ducts, glandular structures and aggregates of neoplastic cholangiocytes (15) (Fig. 2). In fact, patients who have a desmoplastic reaction rich in CAFs have a significantly lower overall survival and a worse disease-free survival than patients with ICC with lower levels of $\alpha$-SMA positivity (16).

Cholangiocarcinoma 2-D culture models have been described in the literature $(17,18)$ but they use long-standing CCA cell lines of various biliary tumor cell origins with stromal cells derived from non-cholangiocarcinoma tissues. In 2012, Campbell et al (19) presented their novel 3-D organotypic co-culture model of rat cholangiocarcinoma. The aim of our study was to devise a novel in vitro model by which to study CCA tumor-stroma crosstalk to better investigate the interactions between neoplastic cholangiocytes and stromal fibroblasts. From human CCA surgical samples, human biliary epithelial cells (hBECs) and stromal cells (SCs) were isolated and cultured in selective media. Cultures were maintained and passaged several times, and their purity was assessed by fluorescent immunocytochemistry.

\section{Materials and methods}

Cholangiocarcinoma specimens. Liver tissues from patients undergoing CCA resection (Department of Surgery, Regional Center for HPB Surgery, Treviso Regional Hospital, Treviso, Italy) were collected between 2010 and 2012; a total of 37
Table I. Clinical and biochemical findings of the 10 patients with intrahepatic cholangiocarcinoma enrolled in the study.

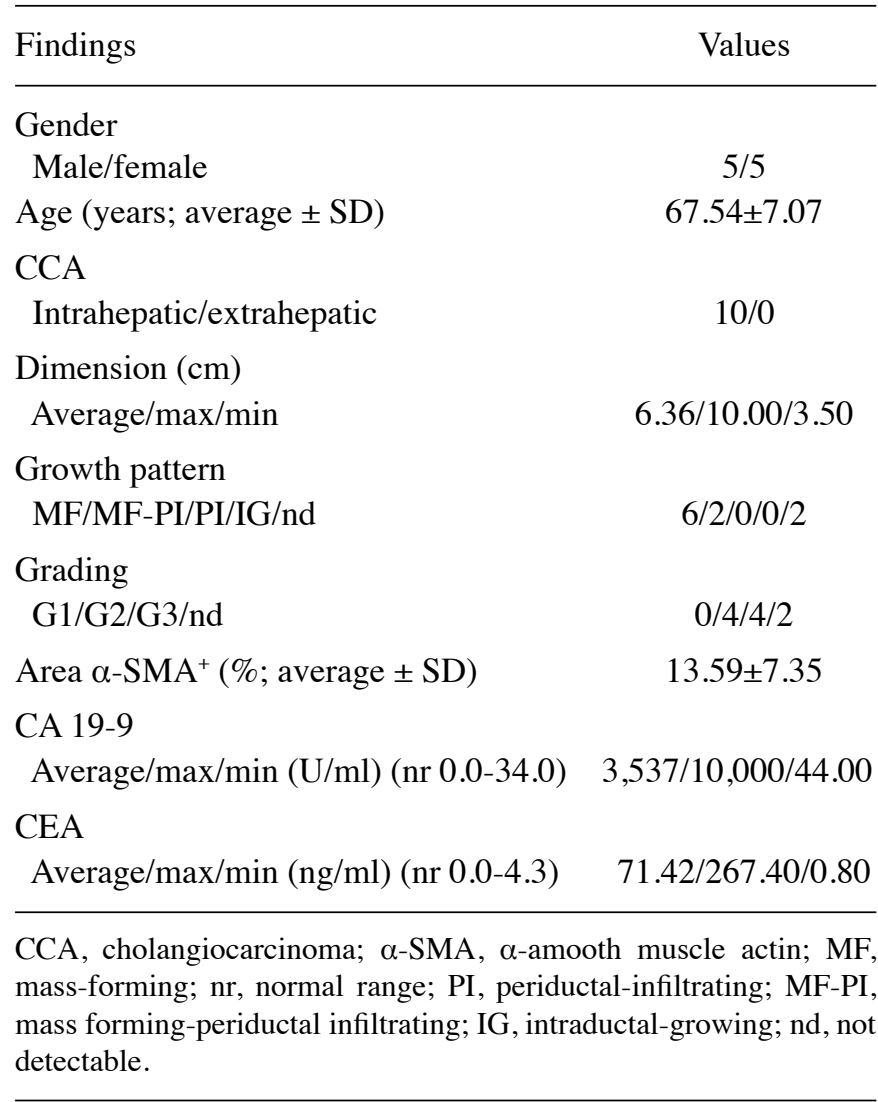

consecutive patients with a diagnosis of primary CCA were managed. Of the total number of patients, 14 were not eligible for surgical resection, 8 were extrahepatic CCA with a mass too low for cell isolation, 2 were not CCA and 3 isolation processes were not successful due to culture infection (Fig. 1). Cultures of biliary and stromal cells were, therefore, obtained from 10 patients and their clinical and biological features are outlined in Table I. According to the classification proposed by the Liver Cancer Study Group of Japan (20), the macroscopic growth patterns were: 6 mass-forming, 2 mass-forming/ periductal-infiltrating and 2 not detectable since they were isolated from metastatic samplings; all were adenocarcinomas (Table I). The study was approved by the local ethical committee of our Institution.

Cell isolation and cultures. CCA cell lines were isolated as described by Fabris et al (21). Briefly, a minimum of $30 \mathrm{~g}$ of liver tissue was finely diced and digested with collagenase type $1 \mathrm{~A}$ ( $1 \mathrm{mg} / \mathrm{ml}$; Sigma Chemical Co.). After a variable incubation time, according to the extent of tissue fibrosis, the liver was sieved through fine mesh (Sigma Screen cup; Sigma Chemical Co.). The homogenate was semi-purified by centrifugation on a 33\%/77\% Percoll (Sigma) gradient to reveal three separate layers: hepatocytes, hBECs and a mixture of blood cells and CAFs. The hBEC layer was extracted while the CAF layer was plated and cultured.

hBECs were immunopurified using an immunomagnetic technique. First, cells were incubated with the monoclonal 
Table II. Characteristics of the primary antibodies used in the immunohistochemistry and immunofluorescence experiments.

\begin{tabular}{llclr}
\hline Antibody & \multicolumn{1}{c}{ Antigenic unmasking } & Dilution & Isotype & Supplier \\
\hline CK7 & $20 \mathrm{~min}$ in citrate, $10 \mathrm{mmol} / \mathrm{l}$ at pH 6.0 & $1: 50$ & Mouse monoclonal & Dako \\
CK19 & $20 \mathrm{~min}$ in citrate, $10 \mathrm{mmol} / \mathrm{l}$ at pH 6.0 & $1: 50$ & Mouse monoclonal & Dako \\
CD68 & $20 \mathrm{~min}$ in citrate, $10 \mathrm{mmol} / \mathrm{l}$ at pH 6.0 & $1: 500$ & Mouse monoclonal & Dako \\
Vimentin & $20 \mathrm{~min}$ in citrate, $10 \mathrm{mmol} / 1$ at $\mathrm{pH} 6.0$ & $1: 100$ & Mouse monoclonal & Dako \\
$\alpha$-SMA & $20 \mathrm{~min}$ in citrate, $10 \mathrm{mmol} / \mathrm{l}$ at $\mathrm{pH} 6.0$ & $1: 100$ & Mouse monoclonal & Dako \\
\hline
\end{tabular}

Table III. Characteristics of the secondary antibodies used in the immunohistochemistry and immunofluorescence experiments.

\begin{tabular}{llcll}
\hline Antibody & Unmasking & Dilution & Isotype & Supplier \\
\hline Alexa Fluor 488 anti-rabbit IgG & Fluorescence & $1: 500$ & Donkey polyclonal & Invitrogen \\
Alexa Fluor 488 anti-mouse IgG & Fluorescence & $1: 500$ & Donkey polyclonal & Invitrogen \\
Alexa Fluor 594 anti-mouse IgG & Fluorescence & $1: 500$ & Goat polyclonal & Invitrogen \\
EnVision anti-mouse & HRP & Net & Goat polyclonal & Dako (K4001) \\
EnVision anti-rabbit & HRP & Net & Goat polyclonal & Dako (K4003) \\
\hline
\end{tabular}

HEA125 antibody (Progen Biotechnik GmBH) and then with IgG1 paramagnetic beads (Dynabeads, Invitrogen). HEA125positive cells were plated and cultured in selective medium. By modifying the Holt's method (10) the remaining cells were plated on flasks, and the medium was replaced after $24 \mathrm{~h}$ to eliminate floating cells in order to leave behind the $\mathrm{SC}$ fraction.

Growth media. CCA cell lines were grown using a selective and complex medium for hBECs and for SCs as described by Fabris et al (21) and Auth et al (22).

Cell lines. In these experiments we used $10 \mathrm{hBEC}$ and $7 \mathrm{SC}$ cell lines obtained from the CCA surgical specimens from the patients with a primary CCA diagnosis managed at Treviso Regional Hospital.

Immunohistochemical analysis of paraffin-embedded tissue sections of CCA. To evaluate $\alpha$-SMA positivity, slides of paraffin-embedded CCA tissue sections $(5-\mu \mathrm{m})$ were used. After deparaffinization, sections were hydrated in alcohol, and endogenous peroxidase activity was quenched for $30 \mathrm{~min}$ in methanol and $10 \%$ hydrogen peroxide $(10 \%)$. Antigen retrieval was performed by heating slides for $20 \mathrm{~min}$ in $10 \mathrm{mM}$ citrate buffer $\mathrm{pH} 6.0$, in a steamer. Sections were incubated overnight at $4^{\circ} \mathrm{C}$ with the primary antibodies listed in Table II. The CCA sections were rinsed with $0.05 \%$ Tween-20 in phosphatebuffered saline (1 M) (PBS) and incubated for $30 \mathrm{~min}$ at room temperature with EnVision anti-mouse antibodies (Dako, Milan, Italy) (Table III). Specimens were developed using 3,3-diaminobenzidine tetrahydrochloride $(0.04 \mathrm{mg} / \mathrm{ml})$ (DAB; Sigma, Milan, Italy) and $\mathrm{H}_{2} \mathrm{O}_{2}(0.01 \%)$ and counterstained with Gill's hematoxylin no. 2 (Sigma). All the antibodies were diluted in PBS (Sigma) supplemented with 5\% normal human serum type 0 and $0.05 \%$ Tween-20 (Sigma).
Immunofluorescence analysis of paraffin-embedded tissue sections of CCA. Double immunofluorescence was performed in selected samples to study the co-localization of the phenotypic markers of hBECs and CAFs. The same methodology from the section above was used up to and including the incubation with the primary antibodies. After 3 washes with PBS plus $0.05 \%$ Tween-20 (Sigma), the samples were incubated with the appropriate fluorescent secondary antibody (Table III) for $30 \mathrm{~min}$. The sections were mounted with Vectashield supplemented with 4.6-diaminidino-2-phenylindole (DAPI; Vector Laboratories) or glycerol supplemented with 5\% 1,4-diazabicyclo(2.2.2)octane (Sigma-Aldrich).

Immunofluorescence of the cell cultures. In the cultured cells, immunoreactivity was detected by immunofluorescence. Briefly, cells were fixed with $4 \%$ paraformaldehyde (Carlo Erba, Milan, Italy) and incubated overnight at $4^{\circ} \mathrm{C}$ with rabbit anti-mouse antibodies (Table II). After washing with PBS, cells were supplemented with $0.05 \%$ Tween-20, and then incubated at room temperature with goat or donkey anti-mouse or antirabbit antibody (Invitrogen, Milan, Italy) (Table II) for $30 \mathrm{~min}$ and mounted with Vectastain + DAPI (Vector Laboratories).

Morphometric analysis. Samples were analyzed with a Nikon Eclipse E800 microscope. Images were captured with a Nikon DS-U1 cooled and analyzed using LuciaG 5.0 (Nikon, Milan, Italy). In the histological samples from resected patients, stromal $\alpha$-SMA positivity was calculated as the positive percentage over the total neoplastic area (calculated in 10 random fields captured at a magnification of x200 for each histological section).

Statistical analysis. Results are reported as averages \pm standard deviation, and data were statistically compared using the Student's t-test. Statistical analysis was performed using the 


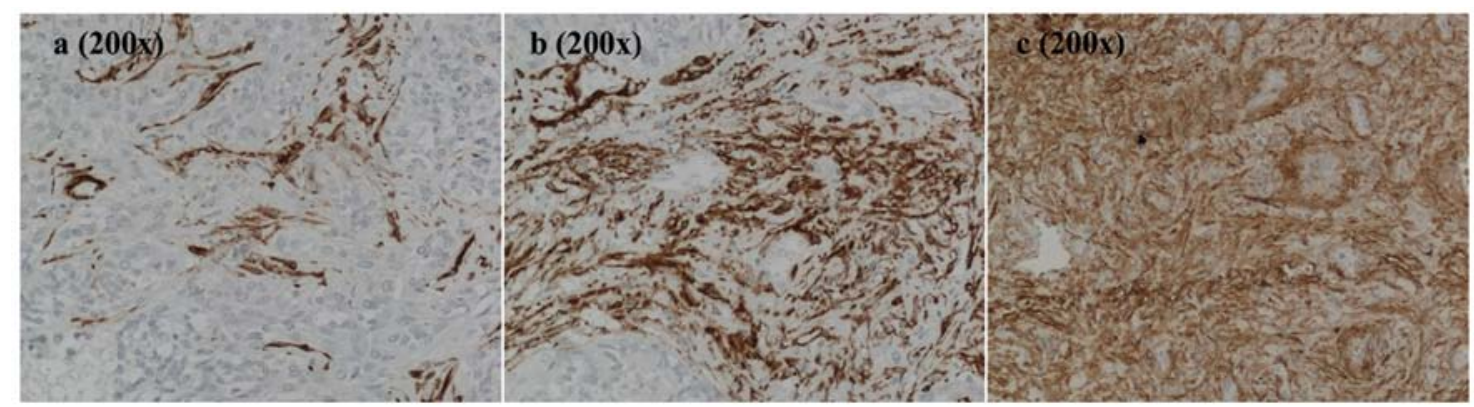

Figure 2. CCA TV\# stromal $\alpha$-SMA for immunohistochemical staining. Samples with (a) low, (b) medium and (c) high percentage of $\alpha$-SMA positivity show a variable degree of stromal fibroblastic components in the liver parenchyma. CCA, cholangiocarcinoma; $\alpha$-SMA, $\alpha$-smooth muscle actin.
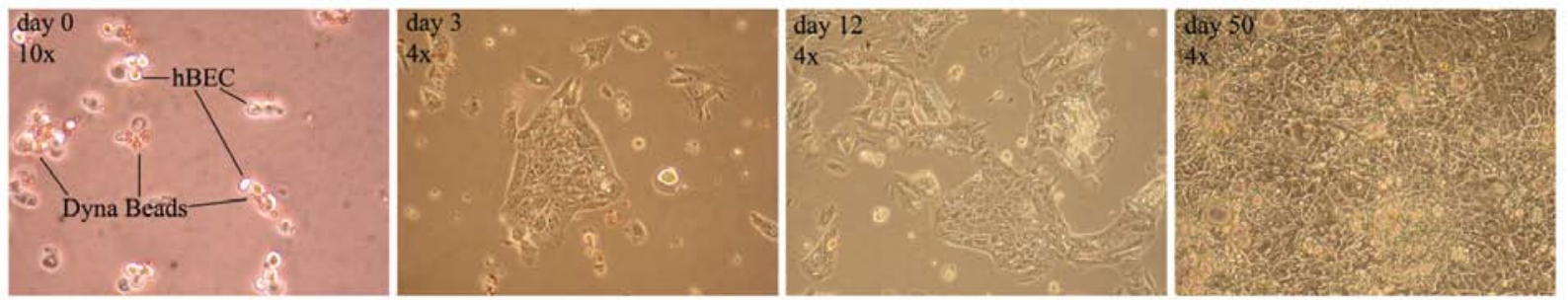

Figure 3. Biliary epithelial cell (hBEC) growth from day 0 to day 50. On day 0, Dynabeads linked to the hBECs.
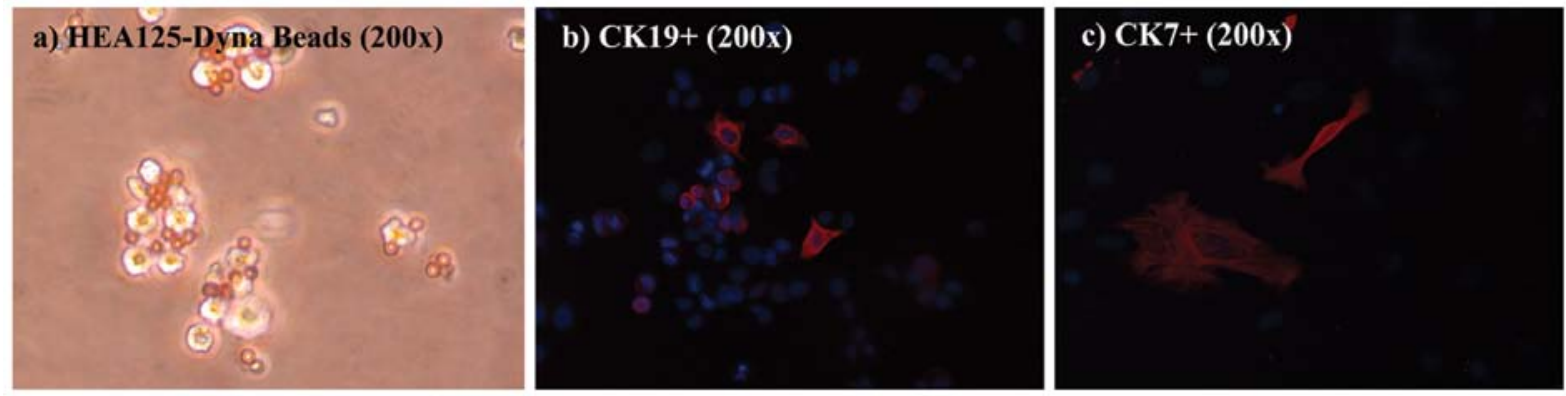

Figure 4. Biliary epithelial cells (hBECs) were positive for (a) HEA125, and at variable degrees, for (b) CK19 and (c) CK7, while they were negative for VMN and $\alpha$-SMA. The nuclear marker DAPI is shown in blue. CK, cytokeratin; VMN, vimentin; $\alpha$-SMA, $\alpha$-smooth muscle actin.

SPSS statistical package (SPSS Inc., Bologna, Italy); the value of significance was set at $\mathrm{P}<0.05$.

\section{Results}

Desmoplastic reaction is increased in cholangiocarcinoma samples. In normal liver parenchyma, fibroblasts are absent with the exception of a few perisinusoidal cells, also known as Ito cells or hepatic stellate cells. CCA, however, presents an increased desmoplastic area characterized by diffuse $\alpha$-SMA positivity.

In samples obtained from the CCA patients, the proportion of desmoplastic reaction was determined using immunohistochemical staining for $\alpha$-SMA (Fig. 2). Samples were characterized by the consistent presence of $\alpha$-SMA positivity at variable degrees. The $\alpha$-SMA-positive area was evaluated by morphometric analysis, and the average distribution of $\alpha$-SMA positivity was $13.59 \pm 7.35 \%$ with a maximum value of $30.08 \pm 0.23 \%$ and a minimum of $5.45 \pm 0.03 \%$ (Table IV). In areas with the lowest percentage (Fig. 2a), nests of hBECs surrounded by SCs were recognized. As the stromal component increased, the desmoplastic reaction typical of CCA became more apparent (Fig. 2b and c).

Generation of primary biliary and stromal cell cultures from specimens of human intrahepatic CCA. In order to generate an in vitro model by which to study tumor-stroma interactions, intra-hepatic CCA hBECs and SCs were purified by modifying the method described by Fabris et al (21).

As shown in Fig. 3, paramagnetic beads conjugated with HEA125 adhered to the cell surface via epithelial glycoprotein 34 , in the purification process. Once placed, the addition of human hepatocyte growth factor to the medium, a strong trophic agent for cholangiocytes, ensured that the cells reached confluence and their classic epithelial palisade shape was recognized.

As shown in Fig. 4, hBECs were positive for HEA125, and the immunomagnetic Dynabeads are shown adhering to the cell surface after plating. In the cell cultures, immunophenotypic staining of hBECs revealed positivity at variable degrees 

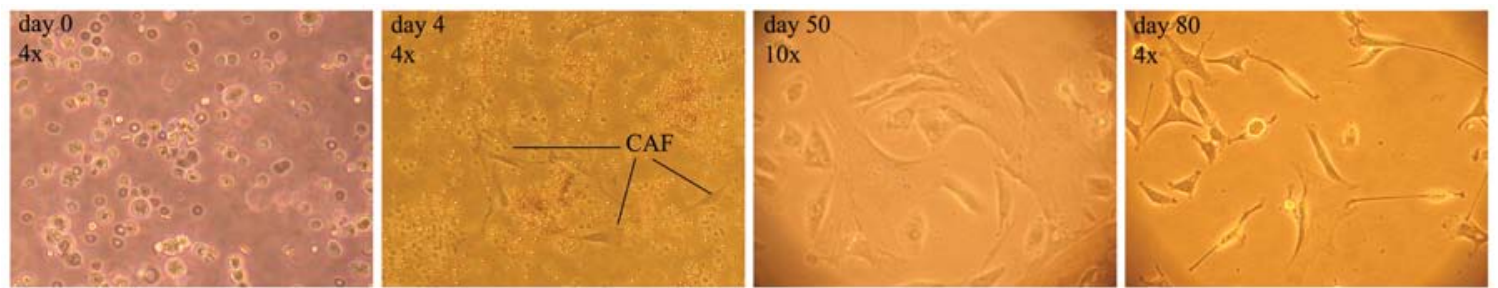

Figure 5. Stromal cell growth from day 0 to day 80 . Selective medium and repeated passages led to the progressive culture purification. Starting from day 4 fibroblasts (CAFs) were morphologically identifiable by the typical spindle shape with multiple cytoplasmic projections that became more evident with cell sorting in the following days.
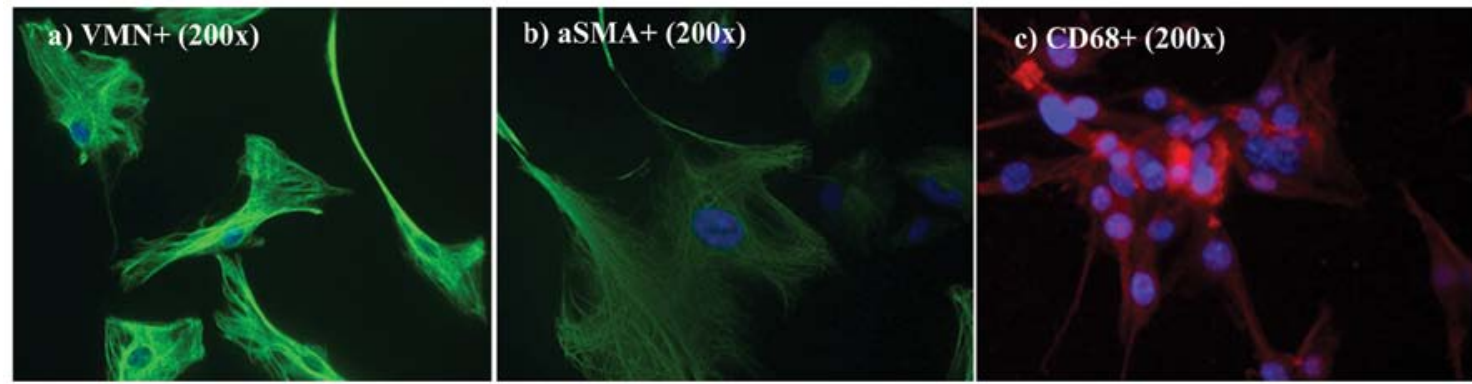

Figure 6. Stromal cell immunophenotypic staining. VMN and $\alpha$-SMA in green (a and b); CD68 in red (c). The nuclear marker DAPI is shown in blue. VMN, vimentin; $\alpha$-SMA, $\alpha$-smooth muscle actin.

Table IV. Values and average distribution of $\alpha$-SMA positivity in the CCA samples (TV\#) collected during the study.

\begin{tabular}{lc}
\hline Patient sample & $\alpha$-SMA positivity \\
\hline TV1 & $10.28 \pm 0.05$ \\
TV2 & $13.04 \pm 0.07$ \\
TV3 & $10.66 \pm 0.07$ \\
TV4 & $5.45 \pm 0.03$ \\
TV6 & $15.43 \pm 0.06$ \\
TV7 & $10.18 \pm 0.08$ \\
TV9 & $30.08 \pm 0.23$ \\
TV10 & $14.37 \pm 0.07$ \\
Average & $13.59 \pm 7.35$ \\
\hline
\end{tabular}

Stromal $\alpha$-SMA positivity was calculated as the $\alpha$-SMA ${ }^{+}$area/total area. $\alpha$-SMA, $\alpha$-smooth muscle actin.

for CK7 and CK19, while hBECs were negative for VMN and $\alpha$-SMA, a profile consistent with their lineage (24).

From the early days of cell culture, isolated SCs were easily detectable by their typical spindle shape, however, they were clouded by a mixture of varying other cell types and debris. The cell culture was progressively purified by the selective growth medium and its replacement after $24 \mathrm{~h}$, and in the following days allowed only for the growth of cells attached to the flask, as illustrated in the timeline of Fig. 5.

To verify the purity of the cultures, immunofluorescence for VMN, a specific fibroblast marker, and $\alpha$-SMA, an activated fibroblast marker, were performed. In Fig. 6a and $b$, cultured cells were positive for both VMN and for $\alpha$-SMA (both green). As previously reported by Kalluri and Zeisberg (7) the lack of specific molecular fibroblast markers is a limiting factor in studying fibroblasts. Even though none are either exclusive to fibroblasts or present in all fibroblasts, VMN and $\alpha$-SMA, intermediate-filament-associated proteins, are specific fibroblast markers $(25,26)$. Nuclei were counterstained with DAPI (blue). As shown in Fig. 6c, only a limited fraction, $<15 \%$, was CD68-positive (CD68-negative fields are not shown here).

\section{Discussion}

CCA is a rare cancer of the bile ducts characterized by an insidious onset and a poor prognosis. The tumor microenvironment has emerged as a key player in CCA invasiveness $(7,11)$. In particular, CAFs are involved in tumorigenesis by promoting the growth and invasion of cancer cells through paracrine communications (14). In addition, CAFs are responsible for the generation of a dense desmoplastic reaction in CCA that frequently prevents surgical resection $(7,12)$.

In tumor samples collected during this study, CAFs were found to be present with a mean percent value of $13.59 \pm 7.35 \%$. In some samples, fibroblasts were detected around neoplastic lobules while, in others, the physiological parenchyma structure was subverted and a widespread fibroblastic parenchymal infiltration was present (Fig. 2).

Between 2010 and 2012, a total of 37 patients with primary CCA were managed at Treviso Hospital (Fig. 1). Of these, 10 intrahepatic CCA specimens were collected. According to the classification proposed by the Liver Cancer Study Group of Japan, mass forming was the predominant macroscopic growth pattern (Table I). 
In order to generate an in vitro model by which to study the interactions between tumoral epithelial and mesenchymal cells in CCA, neoplastic cholangiocytes were isolated by modification of the method described by Fabris et al (21), and stromal fibroblasts by the modification of Holt's method (10). Ten CCA primary biliary epithelial and 7 stromal cell lines were obtained. Both cell types were maintained and passaged several times in culture. Cells were semipurified by centrifugation on Percoll gradient and then hBECs were further immunopurified using anti-HEA125 magnetic beads, and plated and cultured in selective medium. In contrast, SCs were plated and the medium was replaced after $24 \mathrm{~h}$ to eliminate the unattached cells and debris. hBECs and SCs were characterized by immunofluorescence using epithelial (CK7 and CK19) and mesenchymal (VMN, $\alpha$-SMA and CD68) cell markers. hBECs were positive for HEA125, and at variable degrees for CK7 and CK19, while they were negative for VMN and $\alpha$-SMA. SCs were consistently positive for VMN and $\alpha$-SMA, and negative for CK7 and CK19. Only a limited fraction $(<15 \%)$ was positive for CD68.

CCA cells alter the adjacent stroma and create a microenvironment that permits and supports its growth. Previous studies describe long-standing CCA cell lines of various biliary tumor cell origins with stromal cells derived from noncholangiocarcinoma tissues. To our knowledge this is the first report of an in vitro model of primary human CCA cell lines freshly isolated from resected patients. These cell cultures may provide a useful tool to further study the tumor-stroma interactions in human CCA. Future studies will evaluate the migration of stromal cells to epithelium through a Boyden chamber using inhibitors of migration. Furthermore, our epithelial/stroma model will both serve in vitro to investigate the activated pathways and, consequently, to treat these cells with compounds or drugs in order to assess growth and survival under different conditions.

The phenotype and the characterization of the different stromal cells surrounding the CCA stroma have been widely investigated by many authors $(6,7,11,12,22)$, however, this was beyond the specific aim of our study.

\section{References}

1. El-Serag HB and Rudolph KL: Hepatocellular carcinoma: epidemiology and molecular carcinogenesis. Gastroenterology 132 . 2557-2576, 2007.

2. Aljiffry M, Walsh MJ and Molinari M: Advances in diagnosis, treatment and palliation of cholangiocarcinoma: 1990-2009. World J Gastroenterol 15: 4240-4262, 2009.

3. Deoliveira ML, Schulick RD, Nimura Y, Rosen C, Gores G, Neuhaus P and Clavien PA: New staging system and a registry for perihilar cholangiocarcinoma. Hepatology 53: 1363-1371, 2011.

4. Khan SA, Thomas HC, Davidson BR and Taylor-Robinson SD: Cholangiocarcinoma. Lancet 366: 1303-1314, 2005.

5. Sempoux C, Jibara G, Ward SC, Fan C, Qin L, Roayaie S, Fiel MI, Schwartz M and Thung SN: Intrahepatic cholangiocarcinoma: new insights in pathology. Semin Liver Dis 31: 49-60, 2011.

6. Mueller MM and Fusenig NE: Friends or foes - bipolar effects of the tumour stroma in cancer. Nat Rev Cancer 4: 839-849, 2004.

7. Kalluri R and Zeisberg M: Fibroblasts in cancer. Nat Rev Cancer 6: 392-401, 2006.
8. Sirica AE: The role of cancer-associated myofibroblasts in intrahepatic cholangiocarcinoma. Nat Rev Gastroenterol Hepatol 9: 44-54, 2011.

9. Fabris L, Cadamuro M, Moserle L, Dziura J, Cong X, Sambado L, Nardo G, Sonzogni A, Colledan M, Furlanetto A, Bassi N, Massani M, Cillo U, Mescoli C, Indraccolo S, Rugge M, Okolicsanyi L and Strazzabosco M: Nuclear expression of S100A4 calcium-binding protein increases cholangiocarcinoma invasiveness and metastasisation. Hepatology 54: 890-899, 2011.

10. Holt AP, Haughton EL, Lalor PF, Filer A, Buckley CD and Adams DH: Liver myofibroblasts regulate infiltration and positioning of lymphocytes in human liver. Gastroenterology 136: 705-714, 2009.

11. Folkman J: Fundamental concepts of the angiogenic process. Curr Mol Med 3: 643-651, 2003.

12. Albini A and Sporn MB: The tumour microenvironment as a target for chemoprevention. Nat Rev Cancer 7: 139-147, 2007

13. Garin-Chesa P, Old LJ and Rettig WJ: Cell surface glycoprotein of reactive stromal fibroblasts as a potential antibody target in human epithelial cancers. Proc Natl Acad Sci USA 87: 7235-7239, 1990.

14. Xing F, Saidou J and Watabe K: Cancer associated fibroblasts (CAFs) in tumor microenvironment. Front Biosci 15: 166-179, 2010.

15. Sirica AE, Campbell DJ and Dumur CI: Cancer-associated fibroblasts in intrahepatic cholangiocarcinoma. Curr Opin Gastroenterol 27: 276-284, 2011.

16. Nishihara Y, Aishima S, Hayashi A, Iguchi T, Fujita N, Taketomi A, Honda $\mathrm{H}$ and Tsuneyoshi M: CD10 $0^{+}$fibroblasts are more involved in the progression of hilar/extrahepatic cholangiocarcinoma than of peripheral intrahepatic cholangiocarcinoma. Histopathology 55: 423-431, 2009.

17. Chuaysri C, Thuwajit P and Paupairoj A: Alpha-smooth muscle actin-positive fibroblasts promote biliary cell proliferation and correlate with poor survival in cholangiocarcinoma. Oncol Rep 21: 957-969, 2009.

18. Utispan K, Thuwajit $\mathrm{P}$ and Abiko $\mathrm{Y}$ : Gene expression profiling of cholangiocarcinoma-derived fibroblasts reveals alterations related to tumor progression and indicates periostin as a poor prognostic marker. Mol Cancer 9: 13, 2010.

19. Campbell DJW, Dumur CI, Lamour NF, DeWitt JL and Sirica AE: Novel organotypic culture model of cholangiocarcinoma progression. Hepatol Res 42: 1119-1130, 2012.

20. Lim JH: Cholangiocarcinoma: morphologic classification according to growth pattern and imaging findings. AJR Am J Roentgenol 181: 819-827, 2003.

21. Fabris L, Strazzabosco M, Crosby HA, Ballardini G, Hubscher SG, Kelly DA, Neuberger JM, Strain AJ and Joplin R: Characterization and isolation of ductular cells coexpressing neural cell adhesion molecule and Bcl-2 from primary cholangiopathies and ductal plate malformations. Am J Pathol 156: 1599-1612, 2000

22. Auth MK, Woitaschek D, Beste M, Schreiter T, Kim HS, Oppermann E, Joplin RE, Baumann U, Hilgard P, Nadalin S, Markus BH and Blaheta RA: Preservation of the synthetic and metabolic capacity of isolated human hepatocytes by coculture with human biliary epithelial cells. Liver Transpl 11: 410-419, 2011.

23. Cannito S, Novo E, Compagnone A, Valfrè di Bonzo L, Busletta C, Zamara E, Paternostro C, Povero D, Bandino A, Bozzo F, Cravanzola C, Bravoco V, Colombatto S and Parola M: Redox mechanisms switch on hypoxia-dependent epithelialmesenchymal transition in cancer cells. Carcinogenesis 29: 2267-2278, 2008.

24. Bateman AC and Hübscher SG: Cytokeratin expression as an aid to diagnosis in medical liver biopsies. Histopathology 56: 415-425, 2010.

25. Mork C, van Deurs B and Petersen OW: Regulation of vimentin expression in cultured human mammary epithelial cells. Differentiation 43: 146-156, 1990.

26. Tomasek JJ, Gabbiani G, Hinz B, Chaponnier C and Brown RA: Myofibroblasts and mechanoregulation of connective tissue remodelling. Nat Rev Mol Cell Biol 3: 349-363, 2002. 